\section{BMJ Paediatrics Open}

\title{
Prevalence of traumatic psychological stress reactions in children and parents following paediatric surgery: a systematic review and meta-analysis
}

David Paul Turgoose (D , ,,2 Stephanie Kerr, ${ }^{3}$ Paolo De Coppi, ${ }^{3,4}$ Simon Blackburn, ${ }^{3}$ Simon Wilkinson, ${ }^{2}$ Natasha Rooney, ${ }^{2}$ Richard Martin, ${ }^{5}$ Suzanne Gray, ${ }^{2}$ Lee Duncan Hudson (i) 2,6,7

To cite: Turgoose DP, Kerr S, De Coppi P, et al. Prevalence of traumatic psychological stress reactions in children and parents following paediatric surgery: a systematic review and meta-analysis. BMJ Paediatrics Open 2021;5:e001147. doi:10.1136/ bmjpo-2021-001147

Received 28 April 2021 Accepted 7 June 2021
Check for updates

\section{(c) Author(s) (or their} employer(s)) 2021. Re-use permitted under CC BY-NC. No commercial re-use. See rights and permissions. Published by BMJ.

For numbered affiliations see end of article.

\section{Correspondence to} Dr Lee Duncan Hudson; I. hudson@ucl.ac.uk

\section{ABSTRACT}

Background Children undergoing surgery and their parents are at risk of developing post-traumatic stress reactions. We systematically reviewed the literature to understand the prevalence of this issue, as well as potential risk factors.

Methods We conducted a systematic review and metaanalysis, using PubMed, Psyclnfo, Web of Science and Google Scholar, with searches conducted in February 2021. Papers were included if they measured posttraumatic stress in children and/or parents following paediatric surgery and were excluded if they did not use a validated measure of post-traumatic stress. Data were extracted from published reports.

Findings Our search yielded a total of 1672 papers, of which 16 met our inclusion criteria. In meta-analysis, pooled studies of children estimated an overall prevalence of $16 \%$ meeting criteria for post-traumatic stress disorder post surgery ( $\mathrm{N}=187,95 \% \mathrm{Cl} 5 \%$ to $31 \%, \mathrm{I}^{2}=80 \%$ ).

After pooling studies of parents, overall prevalence was estimated at $23 \%\left(\mathrm{~N}=1444,95 \% \mathrm{Cl} 16 \%\right.$ to $\left.31 \%, \mathrm{I}^{2}=91 \%\right)$. Prevalence rates were higher than those reported in the general population. Risk factors reported within studies included length of stay, level of social support and parental mental health.

Interpretation There is consistent evidence of traumatic stress following surgery in childhood which warrants further investigation. Those delivering surgical care to children would benefit from a raised awareness of the potential for post-traumatic stress in their patients and their families, including offering screening and support.

\section{INTRODUCTION}

Traumatic psychological stress reactions can develop in individuals who experience frightening or life-threatening events. ${ }^{1}$ Posttraumatic stress reactions incorporate a range of psychological symptoms. Diagnostic criteria exist to capture these experiences, namely in the form of post-traumatic stress disorder (PTSD) and acute stress disorder (ASD). Symptoms of PTSD and ASD include re-experiencing, avoidance, hyperarousal

\section{What is known about the subject?}

When a child undergoes surgery, the child and/or their parents can experience psychological distress as a result.

- Some children and parents can experience surgery as a traumatic event and subsequently develop symptoms of post-traumatic stress disorder (PTSD).

- There may be risk factors which could help identify families who are more likely to develop such difficulties.

\section{What this study adds?}

The pooled prevalence of PTSD in children undergoing surgery was $16 \%$ and $23 \%$ in parents, which is higher than the general population.

- Cases where the surgery is more complex, or associated health conditions are serious or lifethreatening, appear to increase the risk of PTSD.

- Cases of longer admissions, where the parent reported additional mental health difficulties, or families with less social support were more likely to report PTSD.

and negative alterations in cognitions and mood. ${ }^{2-4}$

Research suggests that lifetime exposure to at least one traumatic event is quite common at around $90 \% .^{5}$ Lifetime prevalence of PTSD in adults has been estimated to be between $6.8 \%{ }^{6}$ to $9.8 \% .^{5}$ In children, studies indicate that $14 \%-43 \%$ of children and adolescents are exposed to at least one traumatic event in their lifetime and that up to $15 \%$ of those exposed go on to develop PTSD, with girls being at higher risk than boys. ${ }^{7}$ It is clear therefore that psychological trauma is a significant issue in both child and adult populations. 
PTSD has been linked to a range of psychological and interpersonal difficulties, including comorbid mental health difficulties such as depression, substance misuse, suicidal ideation, ${ }^{8}$ eating disorders,${ }^{9}$ anxiety disorders ${ }^{10}$ and poor family relationships. ${ }^{11}$ Given that traumatic stress reactions can have such an impact, it is important that services can effectively identify and support families in cases where they may be exposed to trauma. Research is limited, however, and there is a lack of understanding of the risk that surgery poses in terms of the development of post-traumatic stress.

Emerging evidence suggests that children undergoing surgery are at risk of developing traumatic stress reactions post surgery, ${ }^{12-15}$ as are parents of children undergoing surgery. ${ }^{16} 17$ To date, there is only sporadic evidence of, and no consensus on, the rates of traumatic stress development following surgery, or if any risk factors, such as parental stress, ${ }^{18}$ might increase the chances of such difficulties occurring.

An understanding of post-traumatic stress following surgery in childhood is important, to allow for opportunities to mitigate risks or provide appropriate psychological support. Although there is emerging evidence, the literature on this topic has not yet been systematically synthesised. We therefore conducted a systematic review and meta-analysis using Preferred Reporting Items for Systematic Reviews and Meta-Analyses guidance of the literature on: (1) what is the evidence for psychological trauma following surgery in children and their parents and (2) what factors are associated with trauma responses in the same groups.

\section{METHOD}

\section{Search strategy and selection criteria}

We conducted a systematic review with a separate metaanalysis with an overall summary estimate from available data. We searched the literature using PubMed, Web of Science and PsycInfo databases.

We used the following search terms: (Psychological distress OR anxiety OR depression OR mental OR trauma OR traumatic OR post-traumatic stress OR acute stress) AND (children OR adolescent OR family OR sibling OR parent OR carer OR paediatric OR pediatric) AND (surgery OR surgical OR operation OR hospital OR procedure OR anaesthetic). Searches were performed in February 2021 by two independent reviewers who then conferred to agree inclusion and exclusion.

We also performed a separate search using Google Scholar and by searching references of included texts. Google Scholar searches were conducted using the search criteria 'PTSD surgery children' and 'Acute stress disorder surgery children'. We also screened reference lists of included studies for additional papers missed in initial searches.

A priori inclusion criteria were: (1) studies which reported on psychological trauma in children undergoing any type of surgery; (2) studies which reported on psychological trauma in parents of children undergoing surgery; (3) observational or intervention studies and (4) papers in all languages. Exclusion criteria were: (1) studies which did not use a validated measure; (2) studies which reported other mental health variables if there was not an explicit measure of trauma and (3) literature reviews other than systematic reviews.

\section{Data analysis}

Studies were extracted to Endnote which was used to remove duplicates. Quality of studies was assessed using the Newcastle-Ottawa Quality Assessment Scale (NOS), adapted for use with cross-sectional studies. ${ }^{19}$ Studies can achieve an overall rating of a maximum of 10 stars. Each study in the review was assessed by one researcher (SK) scored appropriately. A selection of studies was blindly assessed by another researcher (DPT), with ratings compared and found to match.

\section{Meta-analysis}

Prevalence rates reported in studies for PTSD were pooled in meta-analysis using STATA (V.16) for studies reporting prevalence in: (1) children and (2) parents. The metaprop command was used, with a random effects model using the Freeman-Tukey double arcsine transformation, with pooled estimates and $95 \%$ CIs reported. ${ }^{20}$ Sensitivity analyses were performed by removing studies with greatest bias ( $<5$ total NOS Score). Where available, data were extracted from individual studies to perform meta-regression to identify potential moderator variables on effect sizes across pooled studies.

\section{Patient and public involvement}

Patients and members of the public were not consulted regarding the development of the research question or the research design.

\section{RESULTS}

Initial searches yielded a total of 1671 papers, 609 of which were identified as being duplicates and subsequently removed. The abstracts of the remaining 1062 papers were examined for relevance. In total, 149 papers were then retrieved and reviewed for inclusion. A total of 16 papers were included in the final review. Figure 1 shows a summary of the search and study selection process. Table 1 provides a summary of the studies included.

There was variation in the type of surgery involved in each study, with the most common being cardiac (five studies). In total, six (38\%) studies investigated traumatic stress just in child participants, while eight $(50 \%)$ studies reported data on parents only. A further two (12\%) studies reported on both children and parents concurrently.

Most studies (13) reported whether participants met diagnostic or clinical thresholds for PTSD or ASD, as per the cut-off ranges of the respective measures used. One study gave a breakdown of PTSD symptoms without 


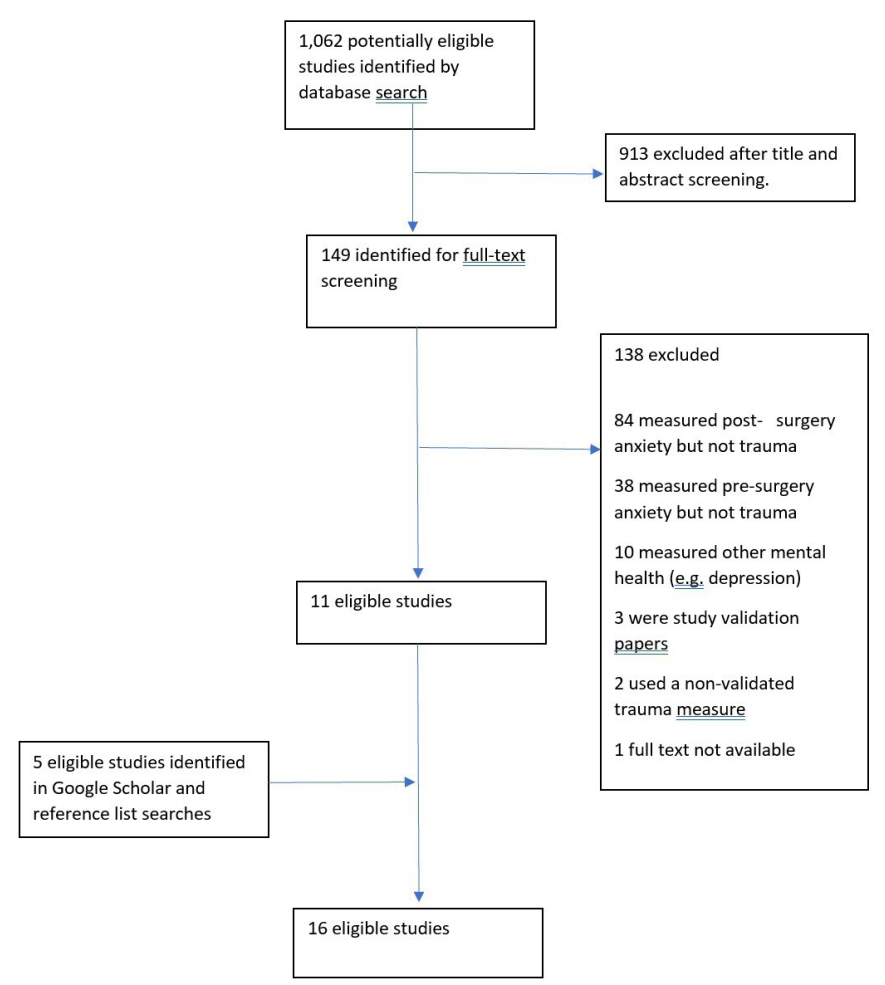

Figure 1 Study selection flowchart.

reporting the number of participants who reached threshold for diagnosable PTSD. The majority of studies (12) provided data regarding correlating or risk factors associated with higher symptoms of traumatic stress.

\section{Prevalence findings in children and young people}

In total, six studies suggested that at least some children had developed PTSD or ASD symptoms after surgery. ${ }^{13-15} 21-23$ One study by Liu $e t a l^{22}$ included participants who had been involved in a natural disaster prior to surgery, which was not controlled for and may have inflated rates of traumatic stress. Another study did not report overall rates of traumatic stress, but did report that high numbers $(56 \%)$ of children displayed difficulties with specific PTSD symptoms, for example, flashbacks. ${ }^{13}$

The four remaining studies which reported overall prevalence of PTSD symptoms at least 3 months post surgery were pooled in meta-analysis (see figure 2) for a total of 187 children. All included studies had a NOS Score $\geq 5$. Heterogeneity was high $\left(\mathrm{I}^{2}=80 \%\right)$. Overall pooled estimate of prevalence was $16 \%$ (95\% CI 5\% to 31\%). Available data from studies allowed meta-regression of type of surgery (cardiac vs other), mean age of children and sex proportion in samples as predictors of prevalence, with none showing significant association (data not shown).

\section{Prevalence findings in parents}

In total, 11 studies reported rates of traumatic stress symptoms in parents. ${ }^{16} 172123-30$ All of these studies reported that at least some parents self-reported symptoms consistent with full PTSD diagnostic criteria. The 11 included studies which reported prevalence of PTSD symptoms at least 3 months post surgery in parents were pooled in meta-analysis for a total of 1444 parents from all studies (see figure 3 ). Heterogeneity was high between studies $\left(\mathrm{I}^{2}=91 \%\right)$. Overall pooled estimate of prevalence of PTSD was $23 \%$ (95\% CI $16 \%$ to $31 \%$ ). Removal of studies with lowest NOS Score did not improve heterogeneity or affect overall pooled estimate significantly. Available data from studies allowed meta-regression of type of surgery, mean age of children and parents, sex proportion of children and parents and parental further education level as predictors of prevalence and none were found to be statistically significant (data not shown).

\section{Findings on potential influencing factors within studies}

Several studies conducted additional analyses to investigate the presence of factors which were associated with or predicted PTSD development in children and parents. A number of variables were reported. Four studies showed that length of stay in hospital was positively associated with PTSD. ${ }^{15-1725}$ Some authors ${ }^{17}$ suggested that this finding was explained by length of stay in hospital reflecting illness severity in the child. Others proposed that children who spend longer in hospital will be exposed to higher levels of accumulative trauma. ${ }^{15}$ This assumes the entire hospital experience to be traumatic, including associated procedures and recovery, over and above the surgery itself. One study which just found this association in fathers suggested that the longer the hospital stay the more parents may struggle to meet the demands of work and family commitments while the child remains in hospital. ${ }^{25}$ This explanation would suggest that the depletion of resources contributes to traumatic stress development or that parents who cannot work due to the caring for a hospitalised child may face financial or other burdens which increase stress and decrease ability to cope.

It is likely that a combination of factors relating to the duration of hospital stay during and post surgery contribute to the development of traumatic stress in children and parents. Most of these risk factors are consistently recorded in clinical care and could easily be used to identify those more in need of additional psychological support.

Several studies reported that different factors relating to the severity of the surgery, complications within the surgery or severity of the child's illness can contribute to the development of traumatic stress. For example, studies reported that more invasive procedures, ${ }^{23} 29$ complications such as the requirement for mechanical ventilation $^{1627}$ or the severity of the child's condition ${ }^{28}$ were all associated with higher PTSD. These findings related to both children and parents.

In three (19\%) studies, associations were found between parental mental health and child traumatic stress. ${ }^{14} 2123$ Specific difficulties that parents reported were PTSD and depression. These findings suggest that the distress and ability to cope of parents can play a significant role in the mental health and traumatic stress of children following surgery. 


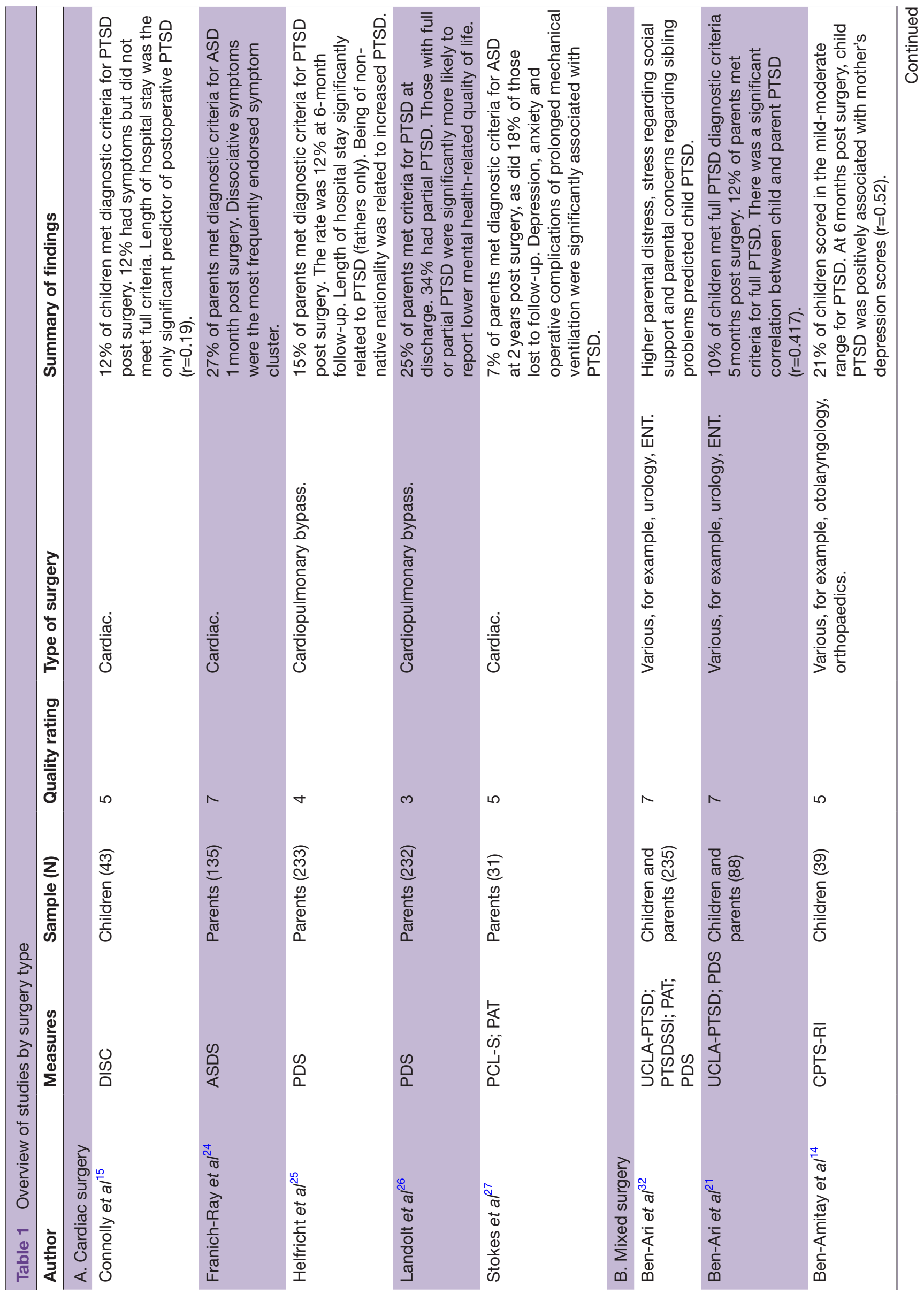




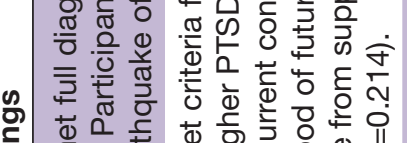
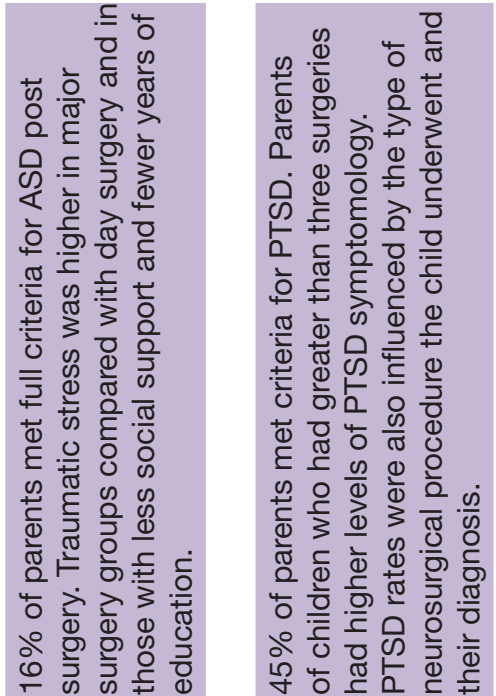

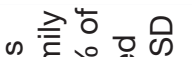

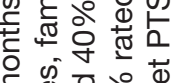

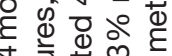
จ की

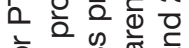

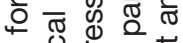

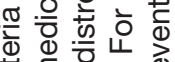

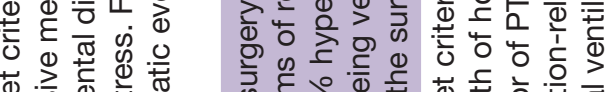
ह क्ष ब क्रें ब

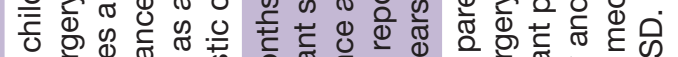

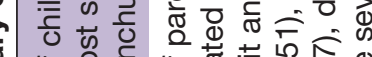
० 造

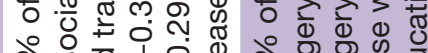

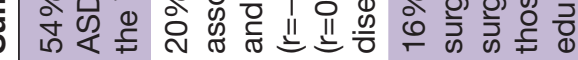

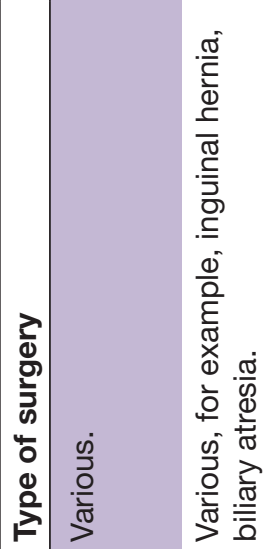

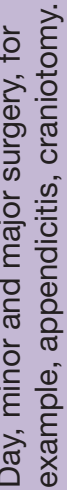

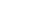

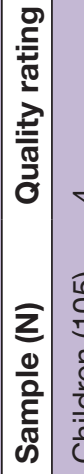

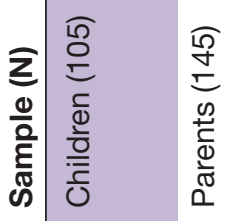

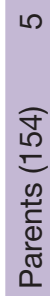

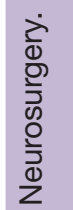

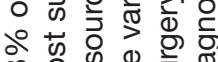

ठ ल

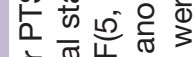

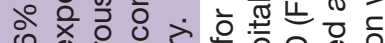

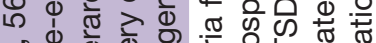

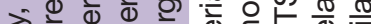
ते

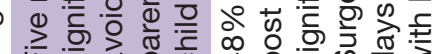

흘

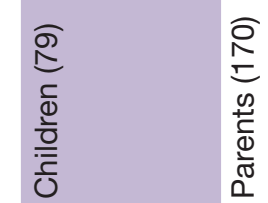

○

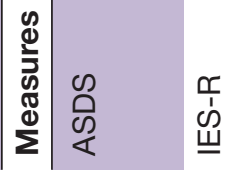

कू

$\frac{1}{1}$

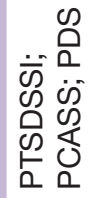
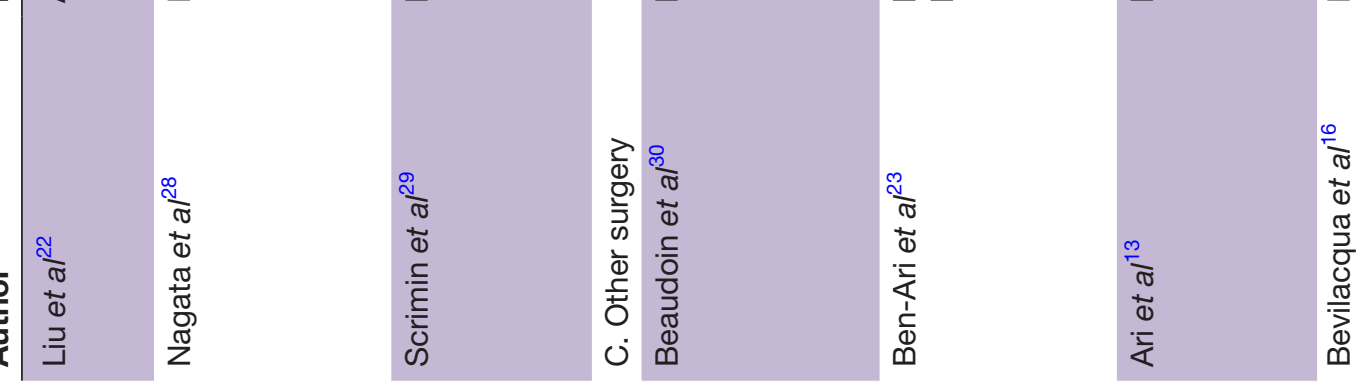


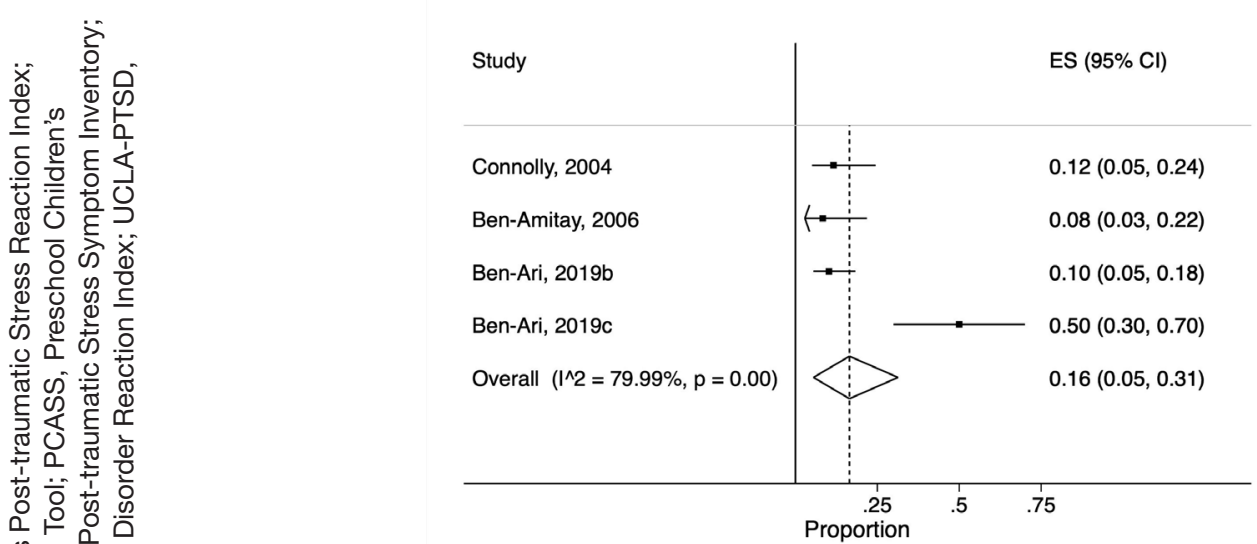

Figure 2 Forest plot of proportions of children meeting criteria for post-traumatic stress disorder $>3$ months after surgery with pooled estimates of overall prevalence with 95\% Cls (with dotted line also showing the pooled overall weighted mean estimate of prevalence $=16 \%$; and $\mathrm{ES}=$ effect size).

The direction of these associations is not necessarily known and could feasibly operate in either direction, that is, parental mental health leads to children being more likely to develop traumatic stress or where children develop traumatic stress, parents are more likely to suffer with their own mental health. These findings are potentially important in helping to identify those who may benefit from additional support.

Some factors relating to social and family support appear to be important when considering traumatic stress following surgery. One study found that when lower levels of family social support were reported using the Psychosocial Assessment Tool II ${ }^{31}$ children were more likely

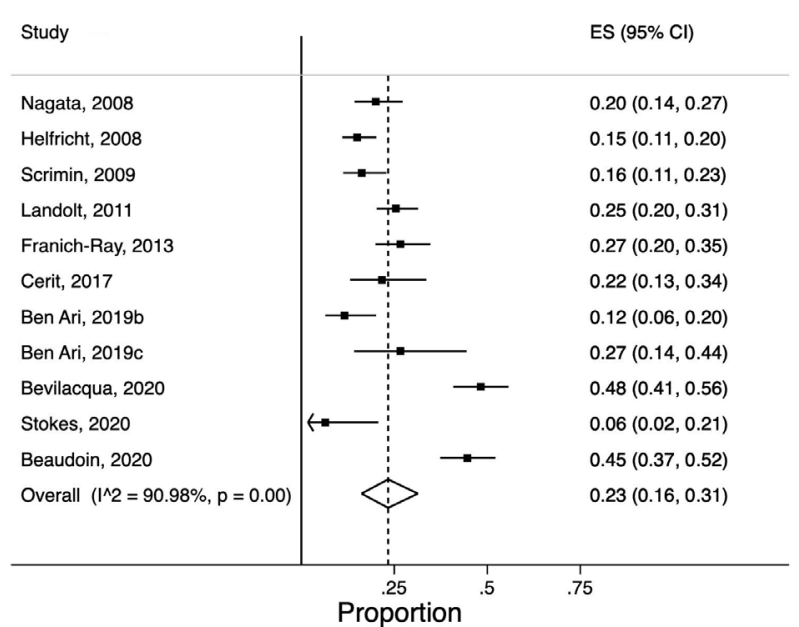

Figure 3 Forest plot of proportions of parents meeting criteria for post-traumatic stress disorder $>3$ months after surgery with pooled estimates of overall prevalence with $95 \% \mathrm{Cls}$ (with dotted line also showing the pooled overall weighted mean estimate of prevalence $=23 \%$; and $\mathrm{ES}=$ effect size). 
to report traumatic stress. ${ }^{32}$ In this study, social support included psychological help, financial support, daily help and help with childcare. Authors suggested that a lack of such resources made it more difficult for children to cope with the stress of the surgery and hospitalisation, not least because it might mean parents were less available to support them. In another study, parents had higher levels of traumatic stress if they reported being far away from their support network or had fewer social contacts around the time of the surgery. ${ }^{28}{ }^{29}$ Additionally, one study found that being a non-native nationality was related to symptom severity and that mothers who used the social services provided by the hospital presented with more severe symptoms of acute PTSD. ${ }^{25}$ Collectively, these findings suggest that social support is an important factor in the development of surgery-related traumatic stress in both children and parents.

\section{DISCUSSION}

This systematic review and meta-analysis of the literature examined post-traumatic stress reactions in children and parents following surgical intervention in a child. In meta-analysis, pooled estimates of prevalence of post-traumatic stress were $16 \%$ in children and $23 \%$ in parents. Past epidemiological research has suggested that $8 \%$ of children in the general population will develop PTSD by 18 years, a figure which rises to $25 \%$ in those who experienced at least one trauma in their lives. ${ }^{33}$ Lower prevalence figures have been reported for adults who have experienced trauma. ${ }^{34}$ This review therefore suggests that paediatric surgery may lead to an increase the risk of PTSD, which has implications for screening and service provision for children undergoing surgery and their families.

We found evidence that a range of psychological and social factors might increase the risk of post-traumatic stress within studies. We did not find evidence for moderating variables in meta-regression once studies were pooled, though there was inconsistent reporting of potential influencing variables across studies thus potentially limiting power. The most commonly reported risk factor in studies was the length of time spent in hospital. This may be explained either by those being in hospital for longer being more exposed to potentially traumatic events, are more unwell, or have had a more serious surgical intervention. It is likely that patients who have experienced complications or multiple procedures are also in this group.

Research suggests that individuals with more social support are less likely to develop chronic PTSD symptoms. ${ }^{35-40}$ There was some evidence to support this in the current review, with several studies reporting a lack of family and social support being associated with traumatic stress in both children and parents. These findings may also relate to duration of hospital stay for children who spend longer in hospital are more likely to miss out on support from extended family or friends; or parents who spend significant amounts of time visiting or staying with a child in hospital may be less able to access social support during this period.

Our search had a number of strengths. The literature search was systematic and robust, including rigorous searches of multiple databases and sources. A priori search terms were used, with agreed inclusion and exclusion criteria for selecting suitable studies. Throughout the search, two researchers were used to cross-reference studies in terms of the inclusion/exclusion criteria, as well as is the quality rating of studies. This will have reduced the risk of error and increased reliability. Our review was necessarily narrow in focus, investigating post-traumatic stress reactions solely. However, children and parents may well present with other mental health (eg, anxiety) or behavioural concerns following surgery, the investigation of which was beyond the scope of this review. In young children particularly, indicators of post-traumatic stress may manifest in ways that are not captured in standard trauma measures, such as via behavioural difficulties. It is important, therefore, that services consider wider aspects of mental health, behaviour and well-being when considering the possible impact of paediatric surgery.

The interpretation of findings is limited by the crosssectional design of studies, meaning that firm conclusions about the cause of traumatic stress in patients are hard to draw. For example, we found that duration of hospital stay was associated with higher levels of traumatic stress. However, there are several associated variables that might explain this, such as whether patients perceive their situation to be life-threatening, ${ }^{23} 2941-43$ which might be more likely in children who spend longer in hospital as they are more likely to have undergone more serious surgery or experienced complications. Further, it was not possible to separate the impact of the surgery specifically from the incident or illness which led the child requiring surgery, which may itself be experienced as traumatic, for example, an accident leading to injury. None of the studies we found controlled for this issue.

None of the studies in the review included a measure of traumatic stress at baseline (before surgery) and again post surgery. This is important as a potential source of bias, since the absence of a baseline measure makes it hard to conclude whether the surgery directly preceded the onset of traumatic stress reactions or whether traumatic stress was already present prior to surgery. Additionally, no studies in the review included control groups against which levels of traumatic stress could be compared. Because we found a number of studies reporting proportions, we were able to perform a metaanalysis. The heterogeneity was high, however we did use a random effects model and performed sensitivity analyses by removing studies with the greatest bias.

Many studies did not have long-term follow-up and only measured traumatic stress directly following surgery. Some research suggests that as many as $56 \%$ of individuals who develop PTSD will spontaneously recover within 5 months of onset ${ }^{10}$ and so findings in the short term may 
not have been maintained. None of the studies included details of whether participants received appropriate interventions for traumatic stress which may have influenced measures at follow-up. Future research in this area should include follow-up measurements to improve our understanding of the long-term persistence and effects of traumatic stress post surgery.

Future studies should include baseline, presurgery measures of trauma symptoms and traumatic event checklists where participants are asked to report types of traumatic events they have experienced. This will help to clarify the role of surgery in the development of traumatic stress reactions. Our study provides evidence of sufficient levels of traumatic stress following paediatric surgery to warrant the evaluation of interventions to support children and families where the child undergoes surgery. Clinical guidelines recommend a period of active monitoring for mild symptoms for a period of 4 weeks, as a way of supporting individuals in the weeks following a trauma. ${ }^{4}$ Future research could investigate report on the implementation of such interventions and on their effectiveness.

\section{CONCLUSIONS}

There is consistent evidence in the literature of posttraumatic stress following surgery in childhood which warrants further investigation. Cases where the surgery is more complex, or the associated health condition is serious or life-threatening, appear to increase the risk of traumatic stress reactions. Several other risk factors have been identified, which may guide services in screening those who may be most at risk. These include cases where the child is in hospital for a longer period, the family are lacking in social support or a parent is suffering with mental health difficulties or high levels of stress.

Those delivering surgical care to children would benefit from a raised awareness of the potential for post-traumatic stress in their patients and their families. Services should also consider using screening measures to help identify families more at risk and to offer support where required. This is particularly relevant in case of service disruptions such as during the current pandemic, where screening for traumatic stress and other mental health difficulties is challenging. Those interventions may provide support to those who report difficulties or are more at risk.

\footnotetext{
Author affiliations

${ }^{1}$ Leeds Institute of Health Sciences, Leeds medical school, University of Leeds, Leeds, UK

${ }^{2}$ Department of Psychology and Mental Health Services, Great Ormond Street Hospital for Children NHS Foundation Trust, London, UK

${ }^{3}$ Department of Specialist and Neonatal Paediatric Surgery, Great Ormond Street Hospital for Children NHS Foundation Trust, London, UK

${ }^{4}$ Developmental Biology and Cancer, Great Ormond Street UCL Institute of Child Health, London, UK

${ }^{5}$ Department of Anaesthethics, Great Ormond Street Hospital for Children NHS Foundation Trust, London, UK

${ }^{6}$ Department of General Paediatrics, Great Ormond Street Hospital for Children NHS Foundation Trust, London, London, UK
}

${ }^{7}$ Population, Policy and Practice Department, Great Ormond Street UCL Institute of Child Health, London, UK

Contributors DPT: conceptualisation, data curation, methodology, formal analysis and writing (original draft). SK: data curation, methodology and formal analysis. LDH: conceptualisation, methodology, formal analysis and writing (review and editing). PDC, SB, NR, RM, SW and SG: writing (review and editing).

Funding The authors have not declared a specific grant for this research from any funding agency in the public, commercial or not-for-profit sectors.

Competing interests None declared.

Patient and public involvement Patients and/or the public were not involved in the design, or conduct, or reporting, or dissemination plans of this research.

Patient consent for publication Not required.

Provenance and peer review Not commissioned; externally peer reviewed.

Data availability statement Data sharing not applicable as no datasets generated and/or analysed for this study.

Open access This is an open access article distributed in accordance with the Creative Commons Attribution Non Commercial (CC BY-NC 4.0) license, which permits others to distribute, remix, adapt, build upon this work non-commercially, and license their derivative works on different terms, provided the original work is properly cited, appropriate credit is given, any changes made indicated, and the use is non-commercial. See: http://creativecommons.org/licenses/by-nc/4.0/.

ORCID iDs

David Paul Turgoose http://orcid.org/0000-0001-5509-9122

Lee Duncan Hudson http://orcid.org/0000-0003-0883-8791

\section{REFERENCES}

1 American Psychiatric Association. Diagnostic and statistical manual of mental disorders. 5th ed. Arlington, VA: Author, 2013.

2 Bisson Jl, Cosgrove S, Lewis C, et al. Post-traumatic stress disorder. BMJ 2015;351:h6161.

3 Dyregrov A, Yule W. A review of PTSD in children. Child Adolesc Ment Health 2006;11:176-84.

4 National Institute for Health and Care Excellence. Guideline 116. post-traumatic stress disorder, 2018. Available: https:// www.nice. org.uk/guidance/ng116 [Accessed Dec 2020].

5 Kilpatrick DG, Resnick HS, Milanak ME, et al. National estimates of exposure to traumatic events and PTSD prevalence using DSM-IV and DSM-5 criteria. J Trauma Stress 2013;26:537-47.

6 Kessler RC, Berglund P, Demler O, et al. Lifetime prevalence and age-of-onset distributions of DSM-IV disorders in the National comorbidity survey replication. Arch Gen Psychiatry 2005;62:593-602.

7 Hamblen J, Barnett E. PTSD in children and adolescents. National Center for PTSD, 2014. Available: www.ncptsd.org

8 Marshall RD, Olfson M, Hellman F, et al. Comorbidity, impairment, and suicidality in subthreshold PTSD. Am J Psychiatry 2001:158:1467-73.

9 Brewerton TD. Eating disorders, trauma, and comorbidity: focus on PTSD. Eat Disord 2007;15:285-304.

10 Galatzer-Levy IR, Nickerson A, Litz BT, et al. Patterns of lifetime PTSD comorbidity: a latent class analysis. Depress Anxiety 2013;30:489-96.

11 Galovski T, Lyons JA. Psychological sequelae of combat violence: A review of the impact of PTSD on the veteran's family and possible interventions. Aggress Violent Behav 2004;9:477-501.

12 Posluszny DM, Edwards RP, Dew MA, et al. Perceived threat and PTSD symptoms in women undergoing surgery for gynecologic cancer or benign conditions. Psychooncology 2011;20:783-7.

13 Ari AB, Peri T, Margalit D, et al. Surgical procedures and pediatric medical traumatic stress (PMTS) syndrome: assessment and future directions. J Pediatr Surg 2018;53:1526-31.

14 Ben-Amitay G, Kosov I, Reiss A, et al. Is elective surgery traumatic for children and their parents? J Paediatr Child Health 2006;42:618-24.

15 Connolly D, McClowry S, Hayman L, et al. Posttraumatic stress disorder in children after cardiac surgery. J Pediatr 2004;144:480-4.

16 Bevilacqua F, Morini F, Ragni B, et al. Pediatric medical traumatic stress (PMTS) in parents of newborns with a congenital anomaly requiring surgery at birth. J Pediatr Surg 2021;56:471-5.

17 Karadeniz Cerit K, Cerit C, Nart Ömer, et al. Post-traumatic stress disorder in mothers of children who have undergone cancer surgery. Pediatr Int 2017;59:996-1001. 
18 Daviss WB, Mooney D, Racusin R, et al. Predicting posttraumatic stress after hospitalization for pediatric injury. J Am Acad Child Adolesc Psychiatry 2000;39:576-83.

19 Modesti PA, Reboldi G, Cappuccio FP, et al. Panethnic differences in blood pressure in Europe: a systematic review and meta-analysis. PLoS One 2016;11:e0147601.

20 Freeman MF, Tukey JW. Transformations related to the angular and the square root. Ann Math Statist. 1950;21:607-11.

21 Ben Ari A, Margalit D, Udassin R, et al. Traumatic stress among school-aged pediatric surgery patients and their parents. Eur $J$ Pediatr Surg 2019;29:437-42.

22 Liu K, Liang X, Guo L, et al. The acute stress disorder in the paediatric surgical children and adolescents injured in the Wenchuan earthquake of China. Stress and Health 2010;26:75-81.

23 Ben-Ari A, Margalit D, Nachshoni L, et al. Traumatic stress among children after surgical intervention for congenital melanocytic nevi: a pilot study. Dermatol Surg 2020;46:e45-52.

24 Franich-Ray C, Bright MA, Anderson V, et al. Trauma reactions in mothers and fathers after their infant's cardiac surgery. $J$ Pediatr Psychol 2013;38:494-505.

25 Helfricht S, Latal B, Fischer JE, et al. Surgery-Related posttraumatic stress disorder in parents of children undergoing cardiopulmonary bypass surgery: a prospective cohort study. Pediatr Crit Care Med 2008;9:217-23.

26 Landolt MA, Buechel EV, Latal B. Predictors of parental quality of life after child open heart surgery: a 6-month prospective study. $J$ Pediatr 2011;158:37-43.

27 Stokes JR, Muscara F, Zannino D, et al. Surgical and psychosocial predictors of mental health in parents of children with cardiac admissions. Ann Thorac Surg 2020;110:1677-82.

28 Nagata S-ichi, Funakosi S, Amae S, et al. Posttraumatic stress disorder in mothers of children who have undergone surgery for congenital disease at a pediatric surgery department. J Pediatr Surg 2008:43:1480-6.

29 Scrimin S, Haynes M, Altoè G, et al. Anxiety and stress in mothers and fathers in the $24 \mathrm{~h}$ after their child's surgery. Child Care Health Dev 2009;35:227-33.

30 Beaudoin W, Moore H, Bliss L, et al. Prevalence of post-traumatic stress disorder in caregivers of pediatric neurosurgical patients. Childs Nerv Syst 2021;37:959-67.

31 Pai ALH, Patiño-Fernández AM, McSherry M, et al. The psychosocial assessment tool (PAT2.0): psychometric properties of a screener for psychosocial distress in families of children newly diagnosed with cancer. J Pediatr Psychol 2008;33:50-62.

32 Ben-Ari A, Benarroch F, Sela Y, et al. Risk factors for the development of medical stress syndrome following surgical intervention. J Pediatr Surg 2020;55:1685-90.

33 Lewis SJ, Arseneault L, Caspi A, et al. The epidemiology of trauma and post-traumatic stress disorder in a representative cohort of young people in England and Wales. Lancet Psychiatry 2019;6:247-56.

34 Sareen J. Posttraumatic stress disorder in adults: impact, comorbidity, risk factors, and treatment. Can J Psychiatry 2014;59:460-7

35 Brewin CR, Andrews B, Valentine JD. Meta-analysis of risk factors for posttraumatic stress disorder in trauma-exposed adults. $J$ Consult Clin Psychol 2000;68:748-66.

36 Ehlers A, Clark DM. A cognitive model of posttraumatic stress disorder. Behav Res Ther 2000;38:319-45.

37 Holeva V, Tarrier N, Wells A. Prevalence and predictors of acute stress disorder and PTSD following road traffic accidents: thought control strategies and social support. Behav Ther 2001;32:65-83.

38 Ozer EJ, Best SR, Lipsey TL, et al. Predictors of posttraumatic stress disorder and symptoms in adults: a meta-analysis. Psychol Bull 2003;129:52-73.

39 Robinaugh DJ, McNally RJ. Trauma centrality and PTSD symptom severity in adult survivors of childhood sexual abuse. $J$ Trauma Stress 2011;24:483-6.

40 Simon N, Roberts NP, Lewis CE, et al. Associations between perceived social support, posttraumatic stress disorder (PTSD) and complex PTSD (CPTSD): implications for treatment. Eur J Psychotraumatol 2019;10:1573129.

41 Jakob JMD, Lamp K, Rauch SAM, et al. The impact of trauma type or number of traumatic events on PTSD diagnosis and symptom severity in treatment seeking veterans. J Nerv Ment Dis 2017;205:83-6.

42 Price M, Szafranski DD, van Stolk-Cooke K, et al. Investigation of abbreviated 4 and 8 item versions of the PTSD checklist 5 . Psychiatry Res 2016;239:124-30.

43 Swartzman S, Booth JN, Munro A, et al. Posttraumatic stress disorder after cancer diagnosis in adults: a meta-analysis. Depress Anxiety 2017;34:327-39. 